\title{
Fight against COVID-19 but avoid disruption of services for other communicable diseases (CDs) and noncommunicable diseases (NCDs)
}

\author{
Hiroaki Mitsuya ${ }^{1,2, *}$ \\ ${ }^{1 .}$ Research Institute, National Center for Global Health and Medicine, Tokyo, Japan; \\ 2. Experimental Retrovirology Section, HIV and AIDS Malignancy Branch, National Cancer Institute, National Institutes of Health, Maryland, USA.
}

\begin{abstract}
COVID-19 has been a global and grave public health threat. The number of deaths from COVID-19 has already surpassed by far that of fatalities from the top three communicable diseases (CDs): human immunodeficiency virus type 1 (HIV) infection, tuberculosis, and malaria. The toll from COVID-19 is also inevitably surpassing hepatitis toll by the beginning of 2021. Moreover, it should be noted that COVID-19 has seriously impacted health services for noncommunicable diseases (NCDs), such as diabetes, hypertension, cancers, and cardiovascular diseases. The most common reasons for the disruption of health services are cancellations of scheduled treatments, a paucity in public transport and a lack of staff due to reassignment of a number of health professionals to COVID-19 works. It's an utmost import that scientifically and practically innovative and rational ways and actions are taken, so that deaths due to the simple lack of essential services for various CDs and NCDs are prevented.
\end{abstract}

Keywords: SARS-CoV-2, COVID-19, communicable diseases (CDs), noncommunicable diseases (NCDs)

The continuing spread of the infection of SARS-CoV-2, which most likely originated in Wuhan or elsewhere in China and causes severe, acute, and often fatal disease (COVID-19), has been a global and grave public health threat. While the World Health Organization (WHO) reports that around $82 \%$ of infected individuals present mild symptoms and require little or no treatment and current estimates put the fatality rate of COVID-19 around $2.5 \%$ in all inflicted cases, much lower than in SARS, which had a global fatality rate of around $10 \%$. However, such a huge number of people have been infected with highly contagious SARS-CoV-2 and the number of confirmed infected cases has risen to more than 76 million, and consequently, the number of deaths is to reach 1.7 million by the end of December, 2020. The number of deaths from COVID-19 has already surpassed by far that of fatalities from the top three communicable diseases (CDs): human immunodeficiency virus type 1 (HIV) infection, tuberculosis, and malaria $(1,2)$ (Figure 1). The toll of hepatitis still continues to rise; however, the toll from COVID-19 is inevitably surpassing hepatitis toll by the beginning of 2021 (1-4) (Figure 1). These numbers reveal how grim the emergence of a new hardto-treat and highly contagious infectious pathogen can be to mankind.

The nature of SARS-CoV-2 and what it causes in human body were virtually unknown and there were no effective vaccines or treatments for the disease at the beginning of the pandemic, which accounts for the high mortality in various cities, regions, and nations early in the pandemic. However, one can say that our learning curve on the virus and its malicious nature has been very steep and with quick accumulation of our knowledge, we have implemented the ways how to avoid infection including setting physical distancing and wearing masks and personal protective equipment. We have also quickly found that a few previously known drugs such as remdesivir and dexamethasone may mitigate the severity of COVID-19. Administration of reportedly effective vaccines has also begun at the time of this writing.

COVID-19 has gravely impacted economics and financial worldwide, but the initial financial stimuli have been effective and appears to have avoided an otherwise greatly catastrophic economic consequences in many regions and nations. However, it should be noted that COVID-19 has also seriously impacted health services for noncommunicable diseases (NCDs). A survey released by WHO in May 2020, in which 155 countries participated, reported the situations of people living with NCDs such as diabetes, hypertension, cancers, and cardiovascular diseases during the beginning of the COVID-19 calamity (5). The survey revealed that the impact of COVID-19 has been of a wide spectrum, but also low-income regions and nations have been at much greater risk of severe COVID-19-related sicknesses and deaths. Another main finding of the survey was that essential health services had been greatly disrupted in many countries (Figure 2). Fifty-three percent of 


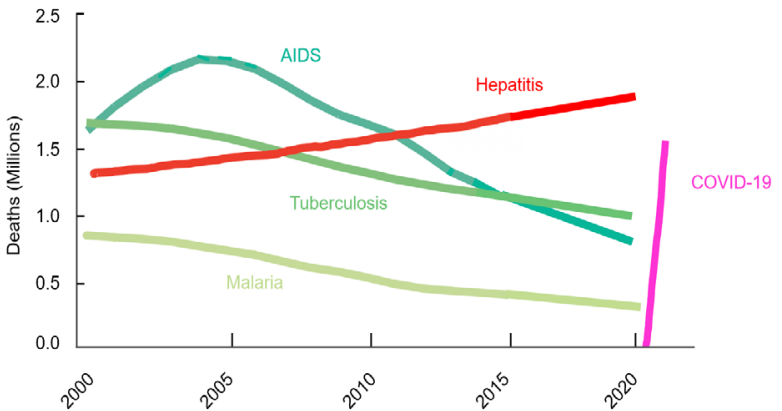

Figure 1. The number of deaths from COVID-19 surpassed by far that of fatalities from the top three communicable diseases (CDs): HIV/AIDS, tuberculosis, and malaria. The death tolls due to HIV/AIDS, tuberculosis, and malaria have been in decline over many years (Data are from WHO: Global health sector strategy on viral hepatitis 2016-2021. Global Burden of Disease and WHO/UNAIDS estimates: https:// ihmeuw.org/3pms, http://ihmeuw.org/3pmt). The number of deaths due to all the viral hepatitis are in rise (https://ihmeuw. org/3pms, http://ihmeuw.org/3pmt); however, the COVID-19 toll is in a sharp rise and will surpass the hepatitis toll very soon, showing how grim the emergence of SARS-CoV-2 is to mankind (https://www.who.int/emergencies/diseases/novelcoronavirus-2019; https://www.worldlifeexpectancy.com/ world-rankings-total-deaths).

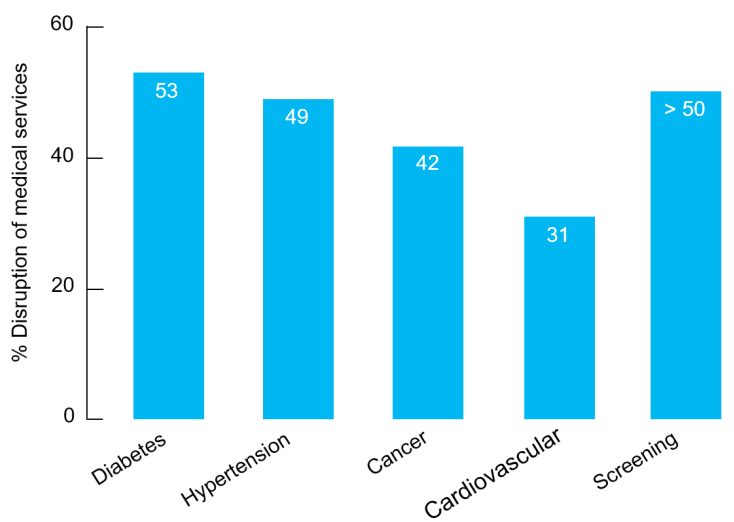

Figure 2. A WHO survey reveals that COVID-19 has greatly disrupted health services for NCDs. A survey by WHO (May 2020), in which 155 countries participated, shows that the COVID-19 pandemic has badly disrupted health services for various NCDs such as diabetes, hypertension, cancers, and cardiovascular diseases. Various screening programs (e.g., for breast and cervical cancers) have also been suspended or postponed in $>50 \%$ of the nations (Data are from https://reliefweb.int/report/world/covid-19-significantlyimpacts-health-services-noncommunicable-diseases).

the nations surveyed reported partial or complete disruption of services for diabetes and diabetes-related complications; $49 \%$ for treatment of hypertension; $42 \%$ for cancer treatment, and $31 \%$ for the response to cardiovascular emergencies. Various screening programs (e.g., for breast and cervical cancers) had also been suspended or postponed in more than $50 \%$ of the nations (Figure 2). One of the major reasons for the disruption of such services was a shortage of therapeutics, diagnostics and medical technologies, but the most common reasons for the disruption of services were cancellations of scheduled treatments, a paucity in public transport

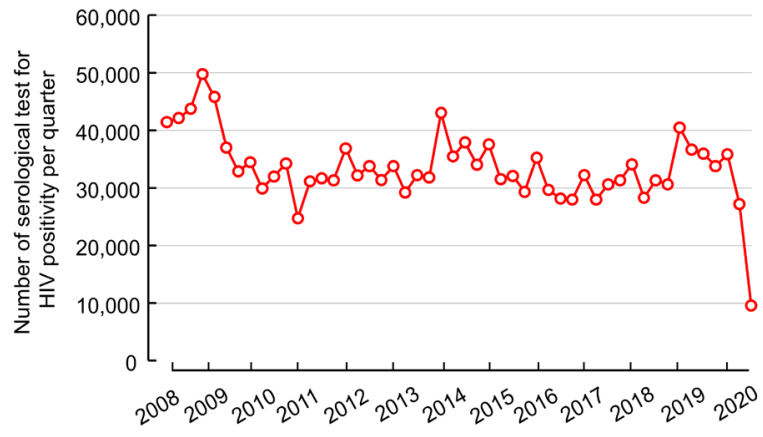

Figure 3. Sudden decrease in the number of serological HIV/AIDS testing in Japan in 2020. The number of serological HIV/AIDS testing had been plateaued counting 30,000 to 40,000 quarterly over the past 12 years; however, that number plunged into only 10,000 in the second quarter of 2020. (Data are from https://api-net.jfap.or.jp/status/japan/ index.html)

and a lack of staff due to reassignment of a number of health professionals to COVID-19 works. Indeed, the survey indicated that in $94 \%$ nations, ministry of health professionals working in the area of NCDs had been partially or fully reassigned to the support of services for COVID-19.

In Japan, we have seen a steep drop $(\sim 70 \%)$ in the number of voluntary screening test for HIV positivity in 2020 (6) (Figure 3). Shin-ichi Oka, Director of AIDS Clinical Center at National Center for Global Health and Medicine, fears that there would be a significant rise in the number of previously healthy people, who did not know their HIV positivity and present frank AIDS, in the upcoming years. Tadao Kakizoe, President of Japan Cancer Society that promotes cancer prevention, guides approximately 11 million cancer screenings, and helps successfully identify as many as 13,000 cancers nationwide every year, warns that the steep decrease by $\sim 30 \%$ in the number of cancer screening that began early March 2020 with the following plummet down to virtually zero would result in a failure to find cancers in $\sim 4,000$ individuals.

It's an utmost import that scientifically and practically innovative and rational ways and actions are taken, by which deaths due to the simple lack of essential services for various CDs and NCDs are prevented. It is likely that our fight against COVID-19 continues much longer than thought in early 2020. It is hoped that the vaccination, which began toward the end of 2020, would work and effective drugs are developed with our knowledge and skills accumulated through our past and on-going dogfights with other viral infections including HIV, hepatitis B virus, hepatitis $\mathrm{C}$ virus, and influenza.

\section{Acknowledgements}

The author thanks Dr. Nobuyo Higashi-Kuwata for discussion and Mses. Kyoko Hattori and Asuka Fujiwara for help. 


\section{Funding: None.}

Conflict of Interest: The author has no conflict of interest to disclose.

\section{References}

1. IHME, GHDx, Viz Hub. Global Both sexes, All ages, 2019, DALYs. https://ihmeuw.org/3pms (accessed December 12, 2020).

2. IHME, GHDx, Viz Hub. Global Both sexes, All ages, 2019, DALYs. http://ihmeuw.org/3pmt (accessed December 12, 2020).

3. World Health Organization. Coronavirus disease (COVID-19) pandemic. https://www.who.int/emergencies/ diseases/novel-coronavirus-2019 (accessed December 13, 2020).

4. World Life Expectancy. World Total Deaths. https://www. worldlifeexpectancy.com/world-rankings-total-deaths (accessed December 13, 2020)

5. ReliefWeb. COVID-19 significantly impacts health services for noncommunicable diseases. https://reliefweb. int/report/world/covid-19-significantly-impacts-healthservices-noncommunicable-diseases (accessed December $15,2020)$.

6. API-Net. Situation in Japan: Committee on AIDS Trends. https://api-net.jfap.or.jp/status/japan/index.html (accessed December 15, 2020) (in Japanese).

Received December 18, 2020; Accepted December 21, 2020.

Released online in J-STAGE as advance publication December 24, 2020.

*Address correspondence to:

Hiroaki Mitsuya, Research Institute, National Center for Global Health and Medicine, 1-21-1 Toyama, Shinjuku-ku, Tokyo162-8655, Japan.

E-mail: hmitsuya@hosp.ncgm.go.jp 